\title{
Thermal [1,5] Hydrogen Sigmatropic Shifts in cis,cis-Cyclononadienes Probed by Gas-Phase Kinetic Studies and Density Functional Theory Calculations
}

\author{
John E. Baldwin, ${ }^{*}, *$ Anuradha S. Raghavan, ${ }^{*}$ B. Andes Hess, Jr., ${ }^{* \dagger}$ and Lidia Smentek ${ }^{\dagger, *}$
}

Departments of Chemistry, Syracuse University, Syracuse, New York 13244, and

Vanderbilt University, Nashville, Tennessee 37235 and Institute of Physics, Nicolaus Copernicus

University, 87-100 Toruń, Poland

E-mail: jbaldwin@syr.edu; b.andes.hess@vanderbilt.edu

\# Syracuse University; $\quad \dagger$ Vanderbilt University; $\quad \ddagger$ Nicolaus Copernicus University

Supporting Information: Four tables of kinetic data, three kinetic plots, photograph of gas phase kinetic setup on vacuum line, calculated geometries and energies of cis,cis-1,3cyclononadiene ground-state conformers, transition structures for conformational interconversions, and transition structures for [1,5] hydrogen shifts (14 files), graphical representations of the 14 structures with bond lengths and bond angles, and a complete reference citation for Gaussian 98W.

S1: $\quad$ Title page and detailed list of contents of the Supporting Information

S2-S3: $\quad$ Tables $1 \mathrm{~S}-4 \mathrm{~S}$, for kinetic data at $240.0,258.3,276.0$, and $287.0^{\circ} \mathrm{C}$

S4-S6: $\quad$ Figures 1S-3S, kinetic plots for data and theory-based functions at 240.0, $258.3^{\circ} \mathrm{C}$, and $276.0^{\circ} \mathrm{C}$

S7: $\quad$ Figure 4S: Gas phase kinetic setup on vacuum line

S8-S21: Calculated geometries and energies for cis,cis-1,3-cyclononadiene groundstate conformations, transition structures for conformational interconversions, and transition structures for [1,5] hydrogen shifts (14 files); (bond distances in $\AA$, angles in degrees)

S22-S24: Graphical representations of the 14 structures with bond lengths and bond angles S25: $\quad$ Complete reference citation for Gaussian $98 \mathrm{~W}$. 
Table 1S. Time-dependent Mole Percent Concentrations of Isomers 1 to 5 at $240.0^{\circ} \mathrm{C}$

\begin{tabular}{cccccc}
\hline Time (s) & $\mathbf{1}$ & $\mathbf{2}$ & $\mathbf{3}$ & $\mathbf{4}$ & $\mathbf{5}$ \\
\hline 0 & 100 & 0 & 0 & 0 & 0 \\
7214 & 52.9 & 27.9 & 14.3 & 4.3 & 0.7 \\
12315 & 45.1 & 26.7 & 18.2 & 7.5 & 2.4 \\
20672 & 36.0 & 22.8 & 24.0 & 13.0 & 4.2 \\
25286 & 29.8 & 21.5 & 24.7 & 14.5 & 9.5 \\
37115 & 23.3 & 18.9 & 25.6 & 19.6 & 12.6 \\
86400 & 14.5 & 12.1 & 26.8 & 24.5 & 22.4 \\
\hline
\end{tabular}

Table 2S. Time-dependent Mole Percent Concentrations of Isomers $\mathbf{1}$ to $\mathbf{5}$ at $258.3^{\circ} \mathrm{C}$

\begin{tabular}{cccccc}
\hline Time (s) & $\mathbf{1}$ & $\mathbf{2}$ & $\mathbf{3}$ & $\mathbf{4}$ & $\mathbf{5}$ \\
\hline 0 & 100 & 0 & 0 & 0 & 0 \\
3661 & 39.4 & 24.8 & 23.9 & 8.2 & 3.8 \\
7328 & 29.5 & 23.3 & 29.0 & 13.6 & 4.6 \\
10821 & 26.9 & 19.7 & 28.9 & 18.7 & 5.9 \\
14450 & 17.5 & 16.5 & 33.5 & 22.2 & 10.3 \\
21646 & 14.8 & 14.1 & 31.4 & 25.5 & 14.2 \\
\hline
\end{tabular}


$-\mathrm{S} 3-$

Table 3S. Time-dependent Mole Percent Concentrations of Isomers $\mathbf{1}$ to $\mathbf{5}$ at $276.0^{\circ} \mathrm{C}$

\begin{tabular}{cccccc}
\hline Time (s) & $\mathbf{1}$ & $\mathbf{2}$ & $\mathbf{3}$ & $\mathbf{4}$ & $\mathbf{5}$ \\
\hline 0 & 100 & 0 & 0 & 0 & 0 \\
1208 & 44.4 & 27.2 & 21.8 & 5.4 & 1.2 \\
2419 & 29.0 & 20.21 & 27.5 & 16.3 & 7.2 \\
3469 & 23.7 & 18.0 & 28.8 & 20.9 & 8.5 \\
7210 & 16.2 & 14.3 & 30.8 & 26.1 & 12.6 \\
11174 & 16.7 & 14.0 & 32.2 & 26.1 & 11.0 \\
19018 & 13.8 & 11.7 & 31.6 & 31.6 & 11.3 \\
\hline
\end{tabular}

Table 4S. Time-Dependent Mole Percent Concentrations of Isomers $\mathbf{1}$ to $\mathbf{5}$ at $287.0{ }^{\circ} \mathrm{C}$

\begin{tabular}{cccccc}
\hline Time (s) & $\mathbf{1}$ & $\mathbf{2}$ & $\mathbf{3}$ & $\mathbf{4}$ & $\mathbf{5}$ \\
\hline 0 & 100 & 0 & 0 & 0 & 0 \\
370 & 54.1 & 26.8 & 14.9 & 4.0 & 0.3 \\
623 & 43.4 & 24.4 & 21.6 & 7.7 & 2.9 \\
911 & 36.8 & 22.2 & 25.4 & 11.6 & 4.0 \\
1528 & 25.1 & 17.7 & 28.9 & 20.2 & 8.1 \\
2710 & 19.5 & 16.4 & 24.9 & 26.8 & 12.4 \\
\hline
\end{tabular}




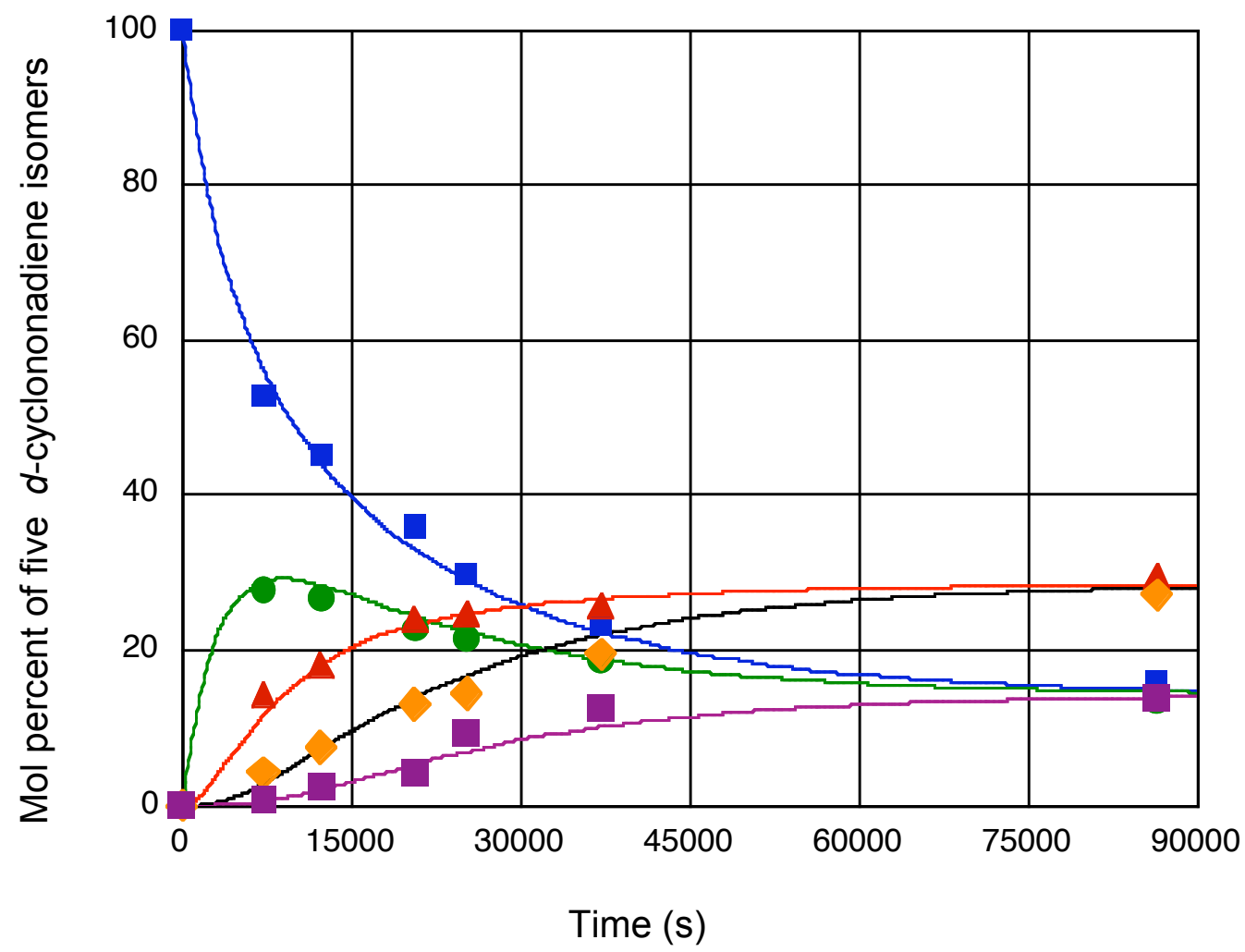

Figure 1S. Kinetic data at $240.0^{\circ} \mathrm{C}$ and theoretical functions for isomers 1 to 5 calculated with the best-fit rate constant $k=5.72 \times 10^{-5} \mathrm{~s}^{-1}$. 


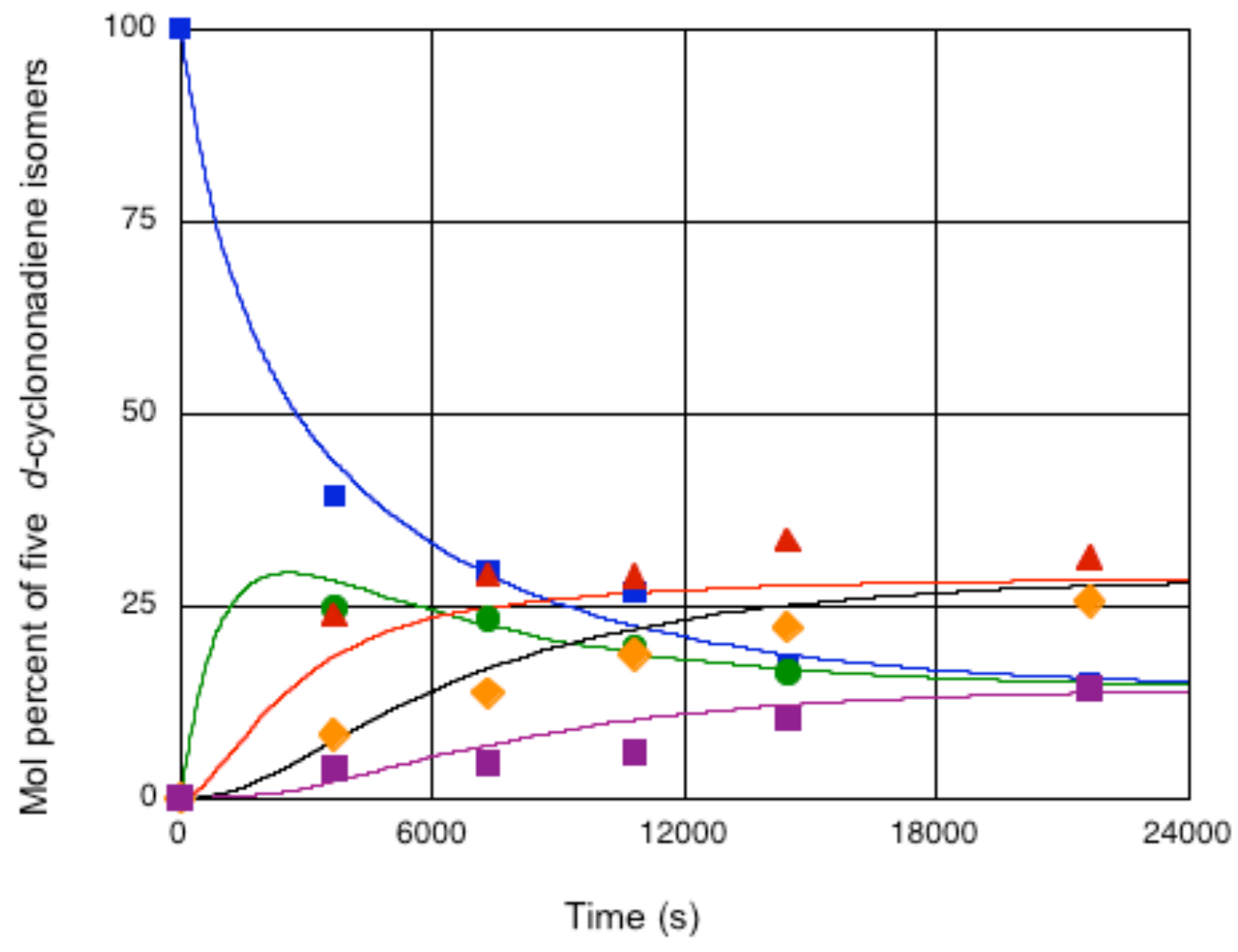

Figure 2S. Kinetic data at $258.3{ }^{\circ} \mathrm{C}$ and theoretical functions for isomers 1 to 5 calculated with the best-fit rate constant $k=1.97 \times 10^{-4} \mathrm{~s}^{-1}$. 


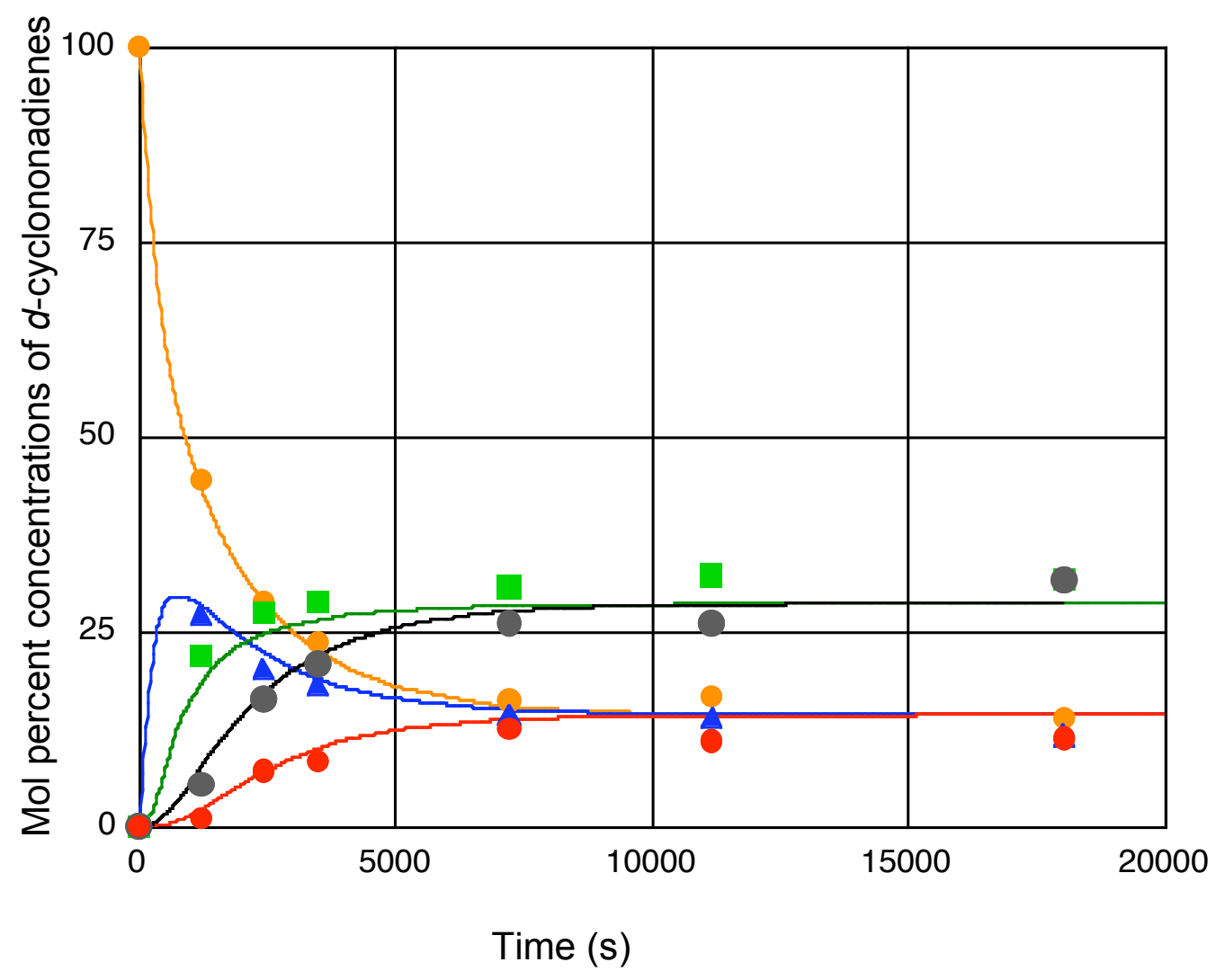

Figure 3S. Kinetic data at $276.0{ }^{\circ} \mathrm{C}$ and theoretical functions for isomers $\mathbf{1}$ to $\mathbf{5}$ calculated with the best-fit rate constant $k=6.01 \times 10^{-4} \mathrm{~s}^{-1}$. 


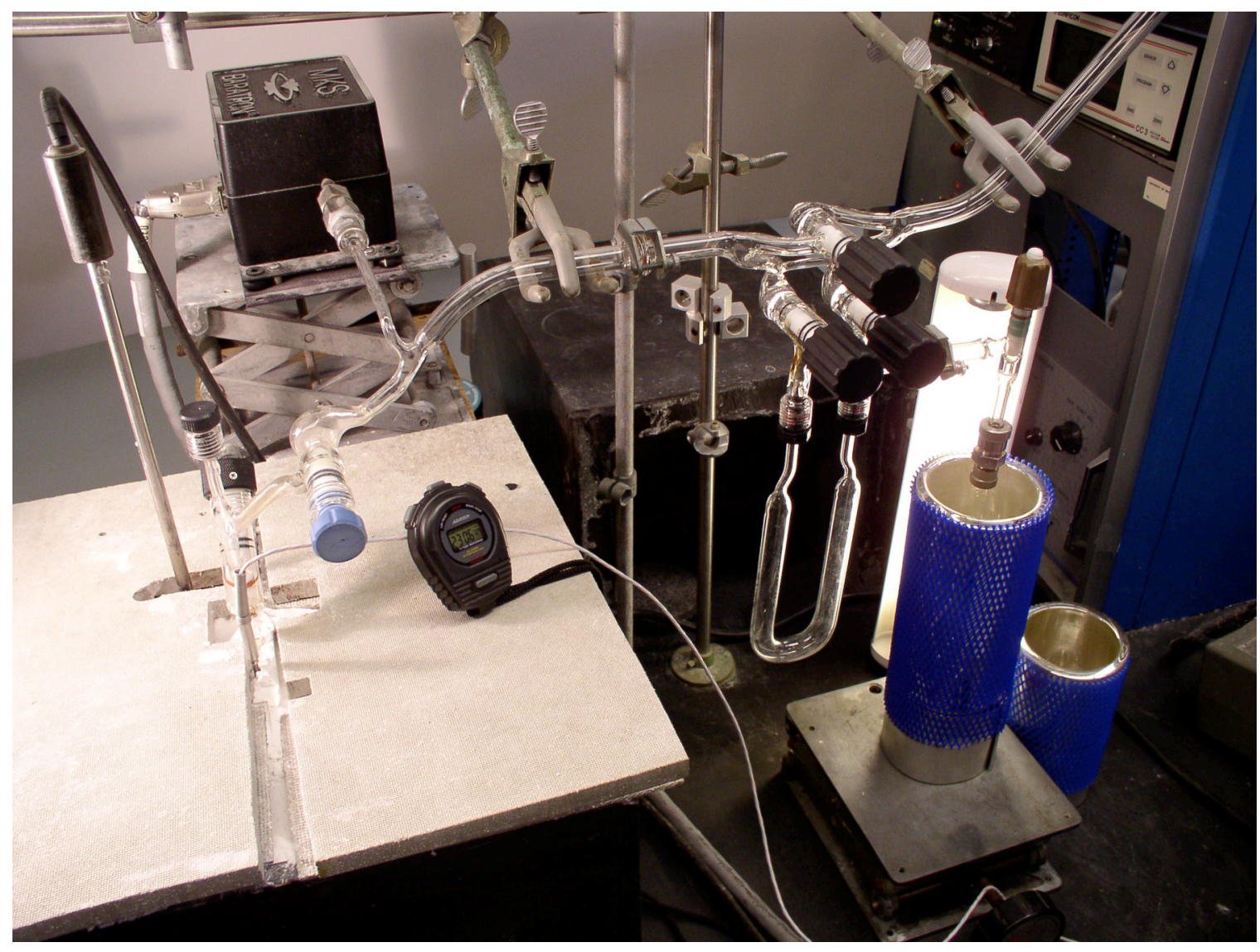

Figure 4S. Gas Phase Kinetic setup on the vacuum line. 
$-\mathrm{S} 8-$

Calculated geometries and energies, for ground-state conformational

isomers:

\begin{tabular}{|c|c|c|c|c|c|}
\hline \multicolumn{6}{|l|}{ C9a } \\
\hline \multicolumn{6}{|c|}{ \# 6-31G* B3LYP FREQ GUESS=READ GEOM=CHECK N } \\
\hline \multicolumn{6}{|c|}{ Standard orientation: } \\
\hline \multirow{2}{*}{$\begin{array}{l}\text { Center } \\
\text { Number }\end{array}$} & \multirow{2}{*}{\multicolumn{2}{|c|}{$\begin{array}{l}\text { Atomic } \\
\text { Number }\end{array}$}} & \multirow{2}{*}{$\begin{array}{l}\text { Atomic } \\
\text { Type }\end{array}$} & \multicolumn{2}{|c|}{ Coordinates (Angstroms } \\
\hline & & & & $\mathrm{X} \quad \mathrm{Y}$ & Y Z Z \\
\hline 1 & 6 & 0 & 1.860421 & 0.693802 & 0.084507 \\
\hline 2 & 6 & 0 & 1.536996 & -0.584710 & 0.813528 \\
\hline 3 & 6 & 0 & 1.065176 & 1.705613 & -0.320397 \\
\hline 4 & 6 & 0 & -0.380819 & 1.981130 & -0.281889 \\
\hline 5 & 6 & 0 & -1.484877 & 1.315615 & 0.119799 \\
\hline 6 & 6 & 0 & -1.674248 & -0.029411 & 0.780149 \\
\hline 7 & 6 & 0 & -0.239553 & -1.413782 & -0.933575 \\
\hline 8 & 6 & 0 & 1.012936 & -1.725626 & -0.098145 \\
\hline 9 & 6 & 0 & -1.561572 & -1.251278 & -0.166730 \\
\hline 10 & 1 & 0 & 2.912439 & 0.806492 & -0.179137 \\
\hline 11 & 1 & 0 & 0.833093 & -0.394780 & 1.624619 \\
\hline 12 & 1 & 0 & 2.453204 & -0.944558 & 1.298856 \\
\hline 13 & 1 & 0 & 1.598733 & 2.508764 & -0.829919 \\
\hline 14 & 1 & 0 & -0.599371 & 2.949022 & -0.734524 \\
\hline 15 & 1 & 0 & -2.418827 & 1.841238 & -0.080655 \\
\hline 16 & 1 & 0 & -0.992197 & -0.148022 & 21.623915 \\
\hline 17 & 1 & 0 & -2.680090 & -0.047149 & 1.218228 \\
\hline 18 & 1 & 0 & -0.374614 & -2.239847 & $7-1.645642$ \\
\hline 19 & 1 & 0 & -0.057442 & -0.519339 & -1.540359 \\
\hline 20 & 1 & 0 & 0.829939 & -2.610554 & 0.528634 \\
\hline 21 & 1 & 0 & 1.819922 & -2.004402 & -0.789409 \\
\hline 22 & 1 & 0 & -1.757428 & -2.157172 & 0.425212 \\
\hline 23 & 1 & 0 & -2.374124 & -1.187814 & $4-0.903303$ \\
\hline
\end{tabular}

SCF Done: $\mathrm{E}(\mathrm{RB}+\mathrm{HF}-\mathrm{LYP})=-351.342434933 \quad$ A.U. after 1 cycles

Frequencies -- $27.4438 \quad 193.1631 \quad 202.4693$

Zero-point vibrational energy 553559.8 (Joules/Mol)

Zero-point correction $=\quad 0.210840($ Hartree/Particle $)$

Thermal correction to Energy $=\quad 0.219391$

Thermal correction to Enthalpy $=\quad 0.220335$

Thermal correction to Gibbs Free Energy $=0.177136$

Sum of electronic and zero-point Energies $=\quad-351.131595$

Sum of electronic and thermal Energies $=\quad-351.123044$

Sum of electronic and thermal Enthalpies $=\quad-351.122100$

Sum of electronic and thermal Free Energies $=\quad-351.165299$ 
-S9-

C9b

\#6-31g* b3lyp freq nopop

Standard orientation:

\begin{tabular}{|c|c|c|c|c|c|}
\hline Center & Atomic & & mic & Coordinate & s (Angstrom \\
\hline 1 & 6 & 0 & 1.096899 & -0.686781 & -1.221254 \\
\hline 2 & 6 & 0 & -0.118158 & -1.160004 & -1.548826 \\
\hline 3 & 6 & 0 & 1.375645 & 0.704156 & -0.728672 \\
\hline 4 & 6 & 0 & -1.911700 & 0.364299 & -0.542663 \\
\hline 5 & 6 & 0 & -1.397150 & -0.433731 & -1.497953 \\
\hline 6 & 6 & 0 & -1.411100 & 0.700333 & 0.848322 \\
\hline 7 & 6 & 0 & -0.566910 & -0.386131 & 1.565572 \\
\hline 8 & 6 & 0 & 0.960828 & -0.154344 & 1.734284 \\
\hline 9 & 6 & 0 & 1.700020 & 0.813341 & 0.785225 \\
\hline 10 & 1 & 0 & 1.954216 & -1.350566 & -1.339291 \\
\hline 11 & 1 & 0 & -0.175969 & -2.166434 & -1.967237 \\
\hline 12 & 1 & 0 & 2.225552 & 1.121616 & -1.286921 \\
\hline 13 & 1 & 0 & -2.874417 & 0.819125 & -0.780040 \\
\hline 14 & 1 & 0 & -2.026050 & -0.589537 & -2.377171 \\
\hline 15 & 1 & 0 & -0.872482 & 1.659061 & 0.828251 \\
\hline 16 & 1 & 0 & -2.303927 & 0.905548 & 1.452985 \\
\hline 17 & 1 & 0 & -0.985217 & -0.526208 & 2.569550 \\
\hline 18 & 1 & 0 & -0.729551 & -1.334069 & 1.047159 \\
\hline 19 & 1 & 0 & 1.158118 & 0.201558 & 2.754316 \\
\hline 20 & 1 & 0 & 1.447084 & -1.135986 & 1.669067 \\
\hline 21 & 1 & 0 & 1.524394 & 1.852395 & 1.093649 \\
\hline 22 & 1 & 0 & 2.774252 & 0.643331 & 0.936211 \\
\hline 23 & 1 & 0 & 0.512853 & 1.332287 & -0.965943 \\
\hline
\end{tabular}

SCF Done: $\mathrm{E}(\mathrm{RB}+\mathrm{HF}-\mathrm{LYP})=-351.337632739$ A.U. after 14 cycles Frequencies -- $56.2336 \quad 137.5665 \quad 204.3492$

Zero-point correction $=\quad 0.210267($ Hartree/Particle $)$

Thermal correction to Energy= $\quad 0.218970$

Thermal correction to Enthalpy= $\quad 0.219915$

Thermal correction to Gibbs Free Energy $=0.176875$

Sum of electronic and zero-point Energies $=\quad-351.127365$

Sum of electronic and thermal Energies $=\quad-351.118662$

Sum of electronic and thermal Enthalpies $=\quad-351.117718$

Sum of electronic and thermal Free Energies $=\quad-351.160758$ 


\section{C9c}

\#6-31g* b3lyp FREQ nopop geom=check guess=read

Input orientation:

\begin{tabular}{|c|c|c|c|c|c|}
\hline Center & Atomic & & tomic & Coordinate & es (Angstrom \\
\hline Number & Numb & & Type & $\begin{array}{ll}X & Y\end{array}$ & $\mathrm{Z}$ \\
\hline 1 & 6 & 0 & 0.210157 & -1.802176 & -0.240330 \\
\hline 2 & 6 & 0 & -0.764259 & -1.300816 & 0.789459 \\
\hline 3 & 6 & 0 & 1.428490 & -1.328271 & -0.548046 \\
\hline 4 & 6 & 0 & 2.162978 & -0.199206 & 0.035142 \\
\hline 5 & 6 & 0 & 1.771708 & 1.037252 & 0.394252 \\
\hline 6 & 6 & 0 & 0.446881 & 1.759739 & 0.315664 \\
\hline 7 & 6 & 0 & -1.938771 & 0.836626 & -0.182254 \\
\hline 8 & 6 & 0 & -2.024490 & -0.668226 & 0.144139 \\
\hline 9 & 6 & 0 & -0.605945 & 1.361479 & -0.758609 \\
\hline 10 & 1 & 0 & -0.137768 & -2.670775 & -0.802585 \\
\hline 11 & 1 & 0 & -0.280695 & -0.601317 & 1.478381 \\
\hline 12 & 1 & 0 & -1.084941 & -2.161329 & 1.393889 \\
\hline 13 & 1 & 0 & 1.996360 & -1.887855 & -1.293852 \\
\hline 14 & 1 & 0 & 3.228697 & -0.402774 & 0.159564 \\
\hline 15 & 1 & 0 & 2.558914 & 1.659421 & 0.823313 \\
\hline 16 & 1 & 0 & -0.022206 & 1.726566 & 1.312396 \\
\hline 17 & 1 & 0 & 0.691926 & 2.821505 & 0.177605 \\
\hline 18 & 1 & 0 & -2.748430 & 1.066777 & -0.887616 \\
\hline 19 & 1 & 0 & -2.166958 & 1.414190 & 0.725333 \\
\hline 20 & 1 & 0 & -2.887374 & -0.824275 & 0.804621 \\
\hline 21 & 1 & 0 & -2.250153 & -1.224998 & -0.776004 \\
\hline 22 & 1 & 0 & -0.831703 & 2.262144 & -1.341897 \\
\hline 23 & 1 & 0 & -0.186167 & 0.644315 & -1.469651 \\
\hline
\end{tabular}

SCF Done: $\mathrm{E}(\mathrm{RB}+\mathrm{HF}-\mathrm{LYP})=-351.340610454 \quad$ A.U. after 1 cycles

Frequencies -- 63.9402

Zero-point correction= 202.0384

Thermal correction to Energy=

0.210166 (Hartree/Particle)

Thermal correction to Enthalpy=

0.218860

Thermal correction to Gibbs Free Energy $=\quad 0.176787$

Sum of electronic and zero-point Energies $=\quad-351.130444$

Sum of electronic and thermal Energies $=\quad-351.121750$

Sum of electronic and thermal Enthalpies $=\quad-351.120806$

Sum of electronic and thermal Free Energies $=\quad-351.163824$ 


\section{C9d}

\#6-31g* b3lyp FREQ nopop geom=check guess=read

Input orientation:

\begin{tabular}{|c|c|c|c|c|c|}
\hline \multirow{2}{*}{$\begin{array}{l}\text { Center } \\
\text { Number }\end{array}$} & \multirow{2}{*}{$\begin{array}{l}\text { Atomic } \\
\text { Number }\end{array}$} & \multirow{2}{*}{\multicolumn{2}{|c|}{$\begin{array}{l}\text { Atomic } \\
\text { Type }\end{array}$}} & \multicolumn{2}{|c|}{ Coordinates (Angstrom } \\
\hline & & & & X $\quad \mathrm{Y}$ & Z \\
\hline 1 & 6 & 0 & 1.492332 & 0.812804 & -0.824147 \\
\hline 2 & 6 & 0 & 1.844333 & 0.045257 & 0.423060 \\
\hline 3 & 6 & 0 & 0.432446 & 1.613140 & -0.998325 \\
\hline 4 & 6 & 0 & -0.627088 & 1.911955 & -0.010731 \\
\hline 5 & 6 & 0 & -1.605748 & 1.125893 & 0.465032 \\
\hline 6 & 6 & 0 & -2.034038 & -0.284525 & 0.115101 \\
\hline 7 & 6 & 0 & 0.015241 & -1.781817 & 0.664198 \\
\hline 8 & 6 & 0 & 1.492376 & -1.459802 & 0.383306 \\
\hline 9 & 6 & 0 & -1.011204 & -1.312348 & -0.401201 \\
\hline 10 & 1 & 0 & 2.188441 & 0.703923 & -1.657331 \\
\hline 11 & 1 & 0 & 1.351625 & 0.500702 & 1.290839 \\
\hline 12 & 1 & 0 & 2.927111 & 0.141319 & 0.585221 \\
\hline 13 & 1 & 0 & 0.353631 & 2.158619 & -1.941889 \\
\hline 14 & 1 & 0 & -0.638787 & 2.950028 & 0.332727 \\
\hline 15 & 1 & 0 & -2.289187 & 1.607573 & 1.166893 \\
\hline 16 & 1 & 0 & -2.523909 & -0.710025 & 1.003348 \\
\hline 17 & 1 & 0 & -2.842845 & -0.192605 & -0.628686 \\
\hline 18 & 1 & 0 & -0.070218 & -2.869184 & 0.788675 \\
\hline 19 & 1 & 0 & -0.250461 & -1.352447 & 1.640549 \\
\hline 20 & 1 & 0 & 2.101591 & -1.973593 & 1.139436 \\
\hline 21 & 1 & 0 & 1.788954 & -1.884607 & -0.586444 \\
\hline 22 & 1 & 0 & -1.587636 & -2.180477 & -0.746228 \\
\hline 23 & 1 & 0 & -0.500204 & -0.922563 & -1.284864 \\
\hline
\end{tabular}

SCF Done: $\mathrm{E}(\mathrm{RB}+\mathrm{HF}-\mathrm{LYP})=-351.342107403 \quad$ A.U. after 1 cycles Frequencies -- $64.7766 \quad 141.7705 \quad 198.4918$

Zero-point correction $=\quad 0.209785($ Hartree/Particle)

Thermal correction to Energy $=\quad 0.218466$

Thermal correction to Enthalpy $=\quad 0.219410$

Thermal correction to Gibbs Free Energy $=0.176517$

Sum of electronic and zero-point Energies $=\quad-351.132322$

Sum of electronic and thermal Energies $=\quad-351.123642$

Sum of electronic and thermal Enthalpies $=\quad-351.122697$

Sum of electronic and thermal Free Energies $=\quad-351.165590$ 
C9e

\#6-31g* b3lyp freq nopop GUESS=READ

Z-Matrix orientation:

\begin{tabular}{|c|c|c|c|c|c|}
\hline \multirow{2}{*}{$\begin{array}{l}\text { Center } \\
\text { Number }\end{array}$} & \multirow{2}{*}{\multicolumn{2}{|c|}{$\begin{array}{l}\text { Atomic } \\
\text { Number }\end{array}$}} & \multirow{2}{*}{$\begin{array}{l}\text { Atomic } \\
\text { Type }\end{array}$} & \multicolumn{2}{|c|}{ Coordinates (Angstroms) } \\
\hline & & & & $\begin{array}{ll}\mathrm{X} & \mathrm{Y}\end{array}$ & $\mathrm{Z}$ \\
\hline 1 & 6 & 0 & 1.513412 & -1.157926 & 0.685086 \\
\hline 2 & 6 & 0 & 2.100162 & -0.130025 & -0.199754 \\
\hline 3 & 6 & 0 & 0.317298 & -1.733671 & 0.484773 \\
\hline 4 & 6 & 0 & 0.479061 & 1.761521 & 0.358563 \\
\hline 5 & 6 & 0 & 1.612906 & 1.104639 & -0.395199 \\
\hline 6 & 6 & 0 & -0.964833 & 1.647731 & -0.221160 \\
\hline 7 & 6 & 0 & -1.840879 & 0.533386 & 0.403886 \\
\hline 8 & 6 & 0 & -1.929172 & -0.799880 & -0.356267 \\
\hline 9 & 6 & 0 & -0.585915 & -1.475377 & -0.692262 \\
\hline 10 & 1 & 0 & 2.126440 & -1.501515 & 1.520803 \\
\hline 11 & 1 & 0 & 3.005311 & -0.417562 & -0.739568 \\
\hline 12 & 1 & 0 & -0.019282 & -2.489253 & 1.196741 \\
\hline 13 & 1 & 0 & 0.726869 & 2.828205 & 0.434338 \\
\hline 14 & 1 & 0 & 2.122439 & 1.735657 & -1.124690 \\
\hline 15 & 1 & 0 & -1.463820 & 2.606104 & -0.032642 \\
\hline 16 & 1 & 0 & -0.929398 & 1.542333 & -1.313779 \\
\hline 17 & 1 & 0 & -1.489819 & 0.342368 & 1.426609 \\
\hline 18 & 1 & 0 & -2.866552 & 0.911595 & 0.508803 \\
\hline 19 & 1 & 0 & -2.547100 & -1.489449 & 0.236652 \\
\hline 20 & 1 & 0 & -2.471203 & -0.643471 & -1.299772 \\
\hline 21 & 1 & 0 & -0.806998 & -2.437502 & -1.178819 \\
\hline 22 & 1 & 0 & -0.049549 & -0.868800 & -1.428436 \\
\hline 23 & 1 & 0 & 0.463328 & 1.378077 & 1.385668 \\
\hline
\end{tabular}

SCF Done: $\mathrm{E}(\mathrm{RB}+\mathrm{HF}-\mathrm{LYP})=-351.345070563$ A.U. after 10 cycles Frequencies -- $99.4152 \quad 132.7700 \quad 182.5366$

Zero-point correction $=\quad 0.209754$ (Hartree/Particle)

Thermal correction to Energy $=\quad 0.218399$

Thermal correction to Enthalpy= $\quad 0.219343$

Thermal correction to Gibbs Free Energy $=\quad 0.176739$

Sum of electronic and zero-point Energies $=\quad-351.135316$

Sum of electronic and thermal Energies $=\quad-351.126672$

Sum of electronic and thermal Enthalpies $=\quad-351.125728$

Sum of electronic and thermal Free Energies $=\quad-351.168332$ 
C9f

\#6-31g* b3lyp freq nopop guess=read geom=check

Input orientation:

\begin{tabular}{|c|c|c|c|c|c|}
\hline \multirow{2}{*}{$\begin{array}{l}\text { Center } \\
\text { Number }\end{array}$} & \multirow{2}{*}{\multicolumn{2}{|c|}{$\begin{array}{l}\text { Atomic } \\
\text { Number }\end{array}$}} & \multirow{2}{*}{$\begin{array}{l}\text { Atomic } \\
\text { Type }\end{array}$} & \multicolumn{2}{|c|}{ Coordinates (Angstroms } \\
\hline & & & & X $\quad Y$ & $\mathrm{Z}$ \\
\hline 1 & 6 & 0 & 0.736476 & -0.032597 & -2.048557 \\
\hline 2 & 6 & 0 & -0.736476 & 0.032597 & -2.048557 \\
\hline 3 & 6 & 0 & 1.541970 & -0.539752 & -1.100193 \\
\hline 4 & 6 & 0 & -1.133346 & 1.184948 & 0.200767 \\
\hline 5 & 6 & 0 & -1.541970 & 0.539752 & -1.100193 \\
\hline 6 & 6 & 0 & -1.288479 & 0.239292 & 1.432592 \\
\hline 7 & 6 & 0 & 0.000000 & 0.000000 & 2.250368 \\
\hline 8 & 6 & 0 & 1.288479 & -0.239292 & 1.432592 \\
\hline 9 & 6 & 0 & 1.133346 & -1.184948 & 0.200767 \\
\hline 10 & 1 & 0 & 1.211122 & 0.338595 & -2.959421 \\
\hline 11 & 1 & 0 & -1.211122 & -0.338595 & -2.959421 \\
\hline 12 & 1 & 0 & 2.616789 & -0.491189 & -1.281845 \\
\hline 13 & 1 & 0 & -1.749307 & 2.080861 & 0.351338 \\
\hline 14 & 1 & 0 & -0.100284 & 1.534934 & 0.117755 \\
\hline 15 & 1 & 0 & -2.616789 & 0.491189 & -1.281845 \\
\hline 16 & 1 & 0 & -2.055172 & 0.630894 & 2.112623 \\
\hline 17 & 1 & 0 & -1.674760 & -0.726407 & 1.087071 \\
\hline 18 & 1 & 0 & 0.180697 & 0.857305 & 2.913718 \\
\hline 19 & 1 & 0 & -0.180697 & -0.857305 & 2.913718 \\
\hline 20 & 1 & 0 & 1.674760 & 0.726407 & 1.087071 \\
\hline 21 & 1 & 0 & 2.055172 & -0.630894 & 2.112623 \\
\hline 22 & 1 & 0 & 1.749307 & -2.080861 & 0.351338 \\
\hline 23 & 1 & 0 & 0.100284 & -1.534934 & 0.117755 \\
\hline
\end{tabular}

SCF Done: $\mathrm{E}(\mathrm{RB}+\mathrm{HF}-\mathrm{LYP})=-351.340855023 \quad$ A.U. after 1 cycles

Frequencies -- $137.0383 \quad 140.3654 \quad 172.3911$

Zero-point correction $=\quad 0.210312$ (Hartree/Particle)

Thermal correction to Energy $=\quad 0.218884$

Thermal correction to Enthalpy $=\quad 0.219829$

Thermal correction to Gibbs Free Energy $=\quad 0.178135$

Sum of electronic and zero-point Energies $=\quad-351.130543$

Sum of electronic and thermal Energies $=\quad-351.121971$

Sum of electronic and thermal Enthalpies $=\quad-351.121026$

Sum of electronic and thermal Free Energies $=\quad-351.162720$ 


\section{Calculated geometries and energies, for transition structures for conformational interconversions:}

\section{TSab}

\#nopop freq 6-31g* b3lyp guess=read geom=check

Input orientation:

\begin{tabular}{|c|c|c|c|c|c|}
\hline \multirow{2}{*}{$\begin{array}{l}\text { Center } \\
\text { Number }\end{array}$} & \multirow{2}{*}{$\begin{array}{l}\text { Atomic } \\
\text { Number }\end{array}$} & \multirow{2}{*}{\multicolumn{2}{|c|}{$\begin{array}{l}\text { Atomic } \\
\text { Type }\end{array}$}} & \multicolumn{2}{|c|}{ Coordinates (Angstroms } \\
\hline & & & & X $\quad Y$ & $\mathrm{Z}$ \\
\hline 1 & 6 & 0 & 1.327434 & -0.905231 & 0.787567 \\
\hline 2 & 6 & 0 & 0.543444 & -0.476089 & 1.791708 \\
\hline 3 & 6 & 0 & 0.836840 & -1.399598 & -0.542858 \\
\hline 4 & 6 & 0 & -1.807016 & -0.019086 & 0.884802 \\
\hline 5 & 6 & 0 & -0.926425 & -0.435078 & 1.814522 \\
\hline 6 & 6 & 0 & -1.605052 & 0.660135 & -0.454992 \\
\hline 7 & 6 & 0 & -0.386490 & 1.609369 & -0.602778 \\
\hline 8 & 6 & 0 & 0.900108 & 1.103326 & -1.323592 \\
\hline 9 & 6 & 0 & 1.027790 & -0.383953 & -1.702420 \\
\hline 10 & 1 & 0 & 2.406794 & -0.901114 & 0.944295 \\
\hline 11 & 1 & 0 & 1.026744 & -0.200568 & 2.730635 \\
\hline 12 & 1 & 0 & 1.366315 & -2.325476 & -0.807182 \\
\hline 13 & 1 & 0 & -0.219780 & -1.663196 & -0.447747 \\
\hline 14 & 1 & 0 & -2.857822 & -0.146964 & 1.148584 \\
\hline 15 & 1 & 0 & -1.359447 & -0.794708 & 2.750504 \\
\hline 16 & 1 & 0 & -1.609153 & -0.087736 & -1.260988 \\
\hline 17 & 1 & 0 & -2.513354 & 1.250674 & -0.631431 \\
\hline 18 & 1 & 0 & -0.736710 & 2.494975 & -1.146025 \\
\hline 19 & 1 & 0 & -0.120290 & 1.966783 & 0.396773 \\
\hline 20 & 1 & 0 & 1.030378 & 1.674514 & -2.252076 \\
\hline 21 & 1 & 0 & 1.761560 & 1.362087 & -0.696599 \\
\hline 22 & 1 & 0 & 0.329775 & -0.638489 & -2.510970 \\
\hline 23 & 1 & 0 & 2.031195 & -0.513555 & -2.129520 \\
\hline
\end{tabular}

\footnotetext{
SCF Done: $\mathrm{E}(\mathrm{RB}+\mathrm{HF}-\mathrm{LYP})=-351.337589700 \quad$ A.U. after 1 cycles

Frequencies -- -66.6656
Zero-point correction $=$ 201.7961

Thermal correction to Energy= 158.3076

Thermal correction to Enthalpy= 0.210183 (Hartree/Particle)

Thermal correction to Gibbs Free Energy $=\quad 0.178229$

Sum of electronic and zero-point Energies $=\quad-351.127407$

Sum of electronic and thermal Energies $=\quad-351.119556$

Sum of electronic and thermal Enthalpies $=\quad-351.118612$

Sum of electronic and thermal Free Energies $=\quad-351.159360$
} 
TSbc

\#nopop freq 6-31g* b3lyp guess=read geom=check

Input orientation:

\begin{tabular}{|c|c|c|c|c|c|}
\hline Center & Atomic & & tomic & oordinate & $\mathrm{ms}$ \\
\hline Number & Number & & Type & $\mathrm{X}$ & Z \\
\hline 1 & 6 & 0 & -1.330277 & 1.163132 & 0.248803 \\
\hline 2 & 6 & 0 & -0.490045 & 1.834248 & -0.558191 \\
\hline 3 & 6 & 0 & -0.918181 & 0.264765 & 1.378292 \\
\hline 4 & 6 & 0 & 1.842530 & 0.782232 & -0.404191 \\
\hline 5 & 6 & 0 & 0.980769 & 1.809479 & -0.540509 \\
\hline 6 & 6 & 0 & 1.606621 & -0.711215 & -0.305533 \\
\hline 7 & 6 & 0 & 0.344873 & -1.264320 & -1.019773 \\
\hline 8 & 6 & 0 & -0.738192 & -1.928300 & -0.141053 \\
\hline 9 & 6 & 0 & -1.187793 & -1.247975 & 1.184604 \\
\hline 10 & 1 & 0 & -2.402194 & 1.303665 & 0.102889 \\
\hline 11 & 1 & 0 & -0.926519 & 2.525673 & -1.281148 \\
\hline 12 & 1 & 0 & -1.463352 & 0.569742 & 2.284122 \\
\hline 13 & 1 & 0 & 0.140660 & 0.431475 & 1.591220 \\
\hline 14 & 1 & 0 & 2.898100 & 1.057255 & -0.394049 \\
\hline 15 & 1 & 0 & 1.435968 & 2.790925 & -0.689032 \\
\hline 16 & 1 & 0 & 1.612126 & -1.014847 & 0.752634 \\
\hline 17 & 1 & 0 & 2.496948 & -1.194862 & -0.727871 \\
\hline 18 & 1 & 0 & 0.665420 & -2.021251 & -1.746687 \\
\hline 19 & 1 & 0 & -0.107609 & -0.466342 & -1.610684 \\
\hline 20 & 1 & 0 & -0.409446 & -2.945377 & 0.111603 \\
\hline 21 & 1 & 0 & -1.618404 & -2.061837 & -0.783272 \\
\hline 22 & 1 & 0 & -0.718824 & -1.760166 & 2.034665 \\
\hline 23 & 1 & 0 & -2.264697 & -1.426326 & 1.300920 \\
\hline
\end{tabular}

SCF Done: $\mathrm{E}(\mathrm{RB}+\mathrm{HF}-\mathrm{LYP})=-351.337367984$ A.U. after 1 cycles Frequencies -- $-84.9030 \quad 143.1819 \quad 199.4078$

Zero-point correction $=\quad 0.210241($ Hartree/Particle)

Thermal correction to Energy $=\quad 0.218089$

Thermal correction to Enthalpy $=\quad 0.219033$

Thermal correction to Gibbs Free Energy $=0.178249$

Sum of electronic and zero-point Energies $=\quad-351.127127$

Sum of electronic and thermal Energies $=\quad-351.119279$

Sum of electronic and thermal Enthalpies $=\quad-351.118335$

Sum of electronic and thermal Free Energies $=\quad-351.159119$ 
TSed

\#6-31 $\mathrm{g}$ * b3lyp freq nopop GUESS=READ GEOM=CHECK

Input orientation:

\begin{tabular}{|c|c|c|c|c|c|}
\hline \multirow{2}{*}{$\begin{array}{l}\text { Center } \\
\text { Number }\end{array}$} & \multirow{2}{*}{$\begin{array}{l}\text { Atomic } \\
\text { Number }\end{array}$} & \multirow{2}{*}{\multicolumn{2}{|c|}{$\begin{array}{l}\text { Atomic } \\
\text { Type }\end{array}$}} & \multicolumn{2}{|c|}{ Coordinates (Angstroms } \\
\hline & & & & $\mathrm{X}$ & $\mathrm{Z}$ \\
\hline 1 & 6 & 0 & 0.841228 & 1.210655 & 1.095678 \\
\hline 2 & 6 & 0 & 1.350889 & -0.202353 & 1.029812 \\
\hline 3 & 6 & 0 & 0.392021 & 1.984011 & 0.094639 \\
\hline 4 & 6 & 0 & 0.297073 & 1.673274 & -1.337132 \\
\hline 5 & 6 & 0 & -0.151736 & 0.586876 & -1.990967 \\
\hline 6 & 6 & 0 & -0.780406 & -0.711762 & -1.542039 \\
\hline 7 & 6 & 0 & -0.716013 & -1.800682 & 0.831293 \\
\hline 8 & 6 & 0 & 0.390955 & -1.203015 & 1.721143 \\
\hline 9 & 6 & 0 & -1.444348 & -0.847485 & -0.142957 \\
\hline 10 & 1 & 0 & 0.846742 & 1.647037 & 2.096229 \\
\hline 11 & 1 & 0 & 1.545685 & -0.498879 & -0.006177 \\
\hline 12 & 1 & 0 & 2.317561 & -0.235910 & 1.552488 \\
\hline 13 & 1 & 0 & 0.122990 & 3.011301 & 0.348078 \\
\hline 14 & 1 & 0 & 0.625065 & 2.496577 & -1.975695 \\
\hline 15 & 1 & 0 & -0.073093 & 0.644154 & -3.078273 \\
\hline 16 & 1 & 0 & -0.032763 & -1.512608 & -1.665749 \\
\hline 17 & 1 & 0 & -1.539229 & -0.945386 & -2.302113 \\
\hline 18 & 1 & 0 & -1.457380 & -2.262069 & 1.497376 \\
\hline 19 & 1 & 0 & -0.293692 & -2.629090 & 0.244036 \\
\hline 20 & 1 & 0 & 0.972697 & -2.033687 & 2.141819 \\
\hline 21 & 1 & 0 & -0.069674 & -0.696836 & 2.580998 \\
\hline 22 & 1 & 0 & -2.449004 & -1.255967 & -0.305402 \\
\hline 23 & 1 & 0 & -1.593880 & 0.134242 & 0.315559 \\
\hline
\end{tabular}

SCF Done: $\mathrm{E}(\mathrm{RB}+\mathrm{HF}-\mathrm{LYP})=-351.340602782$ A.U. after 1 cycles Frequencies -- $-56.3140 \quad 125.4992 \quad 204.3112$

Zero-point correction $=\quad 0.209930($ Hartree/Particle $)$

Thermal correction to Energy= $\quad 0.217817$

Thermal correction to Enthalpy $=\quad 0.218762$

Thermal correction to Gibbs Free Energy $=0.177817$

Sum of electronic and zero-point Energies $=\quad-351.130673$

Sum of electronic and thermal Energies $=\quad-351.122785$

Sum of electronic and thermal Enthalpies $=\quad-351.121841$

Sum of electronic and thermal Free Energies $=\quad-351.162786$ 
TSde

\#nopop freq 6-31g* b3lyp guess=read geom=check

Input orientation:

\begin{tabular}{|c|c|c|c|c|}
\hline Center & Atomic & Atomic & Coordinate & es (Angstron \\
\hline Number & Numbe & Type & $\begin{array}{ll}X & Y\end{array}$ & $\mathrm{Z}$ \\
\hline 1 & 6 & -1.285633 & 1.167320 & -0.707763 \\
\hline 2 & 6 & -1.883989 & 0.169182 & 0.254198 \\
\hline 3 & 6 & -0.178193 & 1.887931 & -0.486767 \\
\hline 4 & 6 & 0.692089 & 1.695076 & 0.698184 \\
\hline 5 & 6 & 1.578080 & 0.695101 & 0.790205 \\
\hline 6 & 6 & 1.913797 & -0.264382 & -0.333200 \\
\hline 7 & 6 & -0.155962 & -1.812019 & 0.406894 \\
\hline 8 & 6 & -1.565093 & -1.324133 & 0.011388 \\
\hline 9 & 6 & 0.990093 & -1.506415 & -0.586047 \\
\hline 10 & 1 & -1.842870 & 1.341466 & -1.629378 \\
\hline 11 & 1 & -1.581610 & 0.421007 & 1.277500 \\
\hline 12 & 1 & -2.976250 & 0.284719 & 0.215102 \\
\hline 13 & 1 & 0.114775 & 2.648528 & -1.213599 \\
\hline 14 & 1 & 0.592013 & 2.398259 & 1.527504 \\
\hline 15 & 1 & 2.153663 & 0.597075 & 1.711011 \\
\hline 16 & 1 & 2.933602 & -0.626938 & -0.158032 \\
\hline 17 & 1 & 1.957891 & 0.313932 & -1.263300 \\
\hline 18 & 1 & -0.221176 & -2.903421 & 0.506945 \\
\hline 19 & 1 & 0.092011 & -1.435581 & 1.407768 \\
\hline 20 & 1 & -2.292065 & -1.902497 & 0.598263 \\
\hline 21 & 1 & -1.756194 & -1.579816 & -1.040843 \\
\hline 22 & 1 & 1.639447 & -2.388862 & -0.632669 \\
\hline 23 & 1 & 0.555634 & -1.413839 & -1.588820 \\
\hline
\end{tabular}

\footnotetext{
SCF Done: $\mathrm{E}(\mathrm{RB}+\mathrm{HF}-\mathrm{LYP})=-351.336410241 \quad$ A.U. after 1 cycles

Frequencies -- -165.1359

$131.8031 \quad 157.5648$

Zero-point correction= 0.209407 (Hartree/Particle)

Thermal correction to Energy= $\quad 0.217427$

Thermal correction to Enthalpy $=\quad 0.218371$

Thermal correction to Gibbs Free Energy $=\quad 0.177131$

Sum of electronic and zero-point Energies $=\quad-351.127004$

Sum of electronic and thermal Energies $=\quad-351.118984$

Sum of electronic and thermal Enthalpies $=\quad-351.118040$

Sum of electronic and thermal Free Energies $=\quad-351.159279$
} 


\section{TSef}

\#nopop freq 6-31g* b3lyp guess=read geom=check

Input orientation:

\begin{tabular}{|c|c|c|c|c|c|}
\hline \multirow{2}{*}{$\begin{array}{l}\text { Center } \\
\text { Number }\end{array}$} & \multirow{2}{*}{\multicolumn{2}{|c|}{$\begin{array}{l}\text { Atomic } \\
\text { Number }\end{array}$}} & \multirow{2}{*}{$\begin{array}{l}\text { Atomic } \\
\text { Type }\end{array}$} & \multicolumn{2}{|c|}{ Coordinates (Angstroms } \\
\hline & & & & $\begin{array}{ll}X & Y\end{array}$ & $\mathrm{Z}$ \\
\hline 1 & 6 & 0 & 1.613610 & 0.960240 & 1.046040 \\
\hline 2 & 6 & 0 & 0.773525 & 2.016865 & 0.455288 \\
\hline 3 & 6 & 0 & 1.757514 & -0.297547 & 0.598138 \\
\hline 4 & 6 & 0 & -1.406538 & 0.719763 & 0.485677 \\
\hline 5 & 6 & 0 & -0.540284 & 1.917557 & 0.195356 \\
\hline 6 & 6 & 0 & -1.740200 & -0.158317 & -0.757924 \\
\hline 7 & 6 & 0 & -1.368970 & -1.641631 & -0.594797 \\
\hline 8 & 6 & 0 & 0.146102 & -2.018151 & -0.470690 \\
\hline 9 & 6 & 0 & 1.207092 & -0.898571 & -0.672324 \\
\hline 10 & 1 & 0 & 2.184996 & 1.251076 & 1.930160 \\
\hline 11 & 1 & 0 & 1.266097 & 2.972523 & 0.266416 \\
\hline 12 & 1 & 0 & 2.397612 & -0.965103 & 1.178144 \\
\hline 13 & 1 & 0 & -2.344327 & 1.070184 & 0.937038 \\
\hline 14 & 1 & 0 & -0.910860 & 0.101222 & 1.240906 \\
\hline 15 & 1 & 0 & -1.041466 & 2.780712 & -0.244645 \\
\hline 16 & 1 & 0 & -2.816580 & -0.107984 & -0.965336 \\
\hline 17 & 1 & 0 & -1.252331 & 0.253328 & -1.649400 \\
\hline 18 & 1 & 0 & -1.903367 & -2.031314 & 0.281732 \\
\hline 19 & 1 & 0 & -1.788384 & -2.180940 & -1.454192 \\
\hline 20 & 1 & 0 & 0.324396 & -2.484840 & 0.505820 \\
\hline 21 & 1 & 0 & 0.347919 & -2.806047 & -1.205707 \\
\hline 22 & 1 & 0 & 2.060186 & -1.344373 & -1.203718 \\
\hline 23 & 1 & 0 & 0.824999 & -0.109688 & -1.325798 \\
\hline
\end{tabular}

SCF Done: $\mathrm{E}(\mathrm{RB}+\mathrm{HF}-\mathrm{LYP})=-351.338816419$ A.U. after 1 cycles Frequencies -- -134.6796 $166.6799 \quad 175.9525$

Zero-point correction $=\quad 0.209985($ Hartree/Particle $)$

Thermal correction to Energy= $\quad 0.217872$

Thermal correction to Enthalpy $=\quad 0.218816$

Thermal correction to Gibbs Free Energy $=0.177915$

Sum of electronic and zero-point Energies $=\quad-351.128831$

Sum of electronic and thermal Energies $=\quad-351.120945$

Sum of electronic and thermal Enthalpies $=\quad-351.120001$

Sum of electronic and thermal Free Energies $=\quad-351.160902$ 


\section{Calculated geometries and energies, for transition structures for [1,5]}

\section{hydrogen shifts:}

\section{TS1}

\#6-31g* b3lyp freq geom=checkpoint guess=read

\begin{tabular}{|c|c|c|c|c|c|}
\hline \multirow{3}{*}{$\begin{array}{l}\text { Center } \\
\text { Number }\end{array}$} & \multicolumn{3}{|c|}{ Input orientation: } & & \\
\hline & Atomic & & Atomic & \multicolumn{2}{|c|}{ Coordinates (Angstroms } \\
\hline & Number & & Type & $\begin{array}{ll}\mathrm{X} & \mathrm{Y}\end{array}$ & Y Z \\
\hline 1 & 6 & 0 & 1.214782 & 1.376956 & -0.568548 \\
\hline 2 & 6 & 0 & 0.000000 & 1.554724 & -1.239888 \\
\hline 3 & 6 & 0 & 1.337120 & 0.665720 & 0.660868 \\
\hline 4 & 6 & 0 & -1.337120 & 0.665720 & 0.660868 \\
\hline 5 & 6 & 0 & -1.214782 & 1.376956 & -0.568548 \\
\hline 6 & 6 & 0 & -1.454088 & -0.866940 & 0.748826 \\
\hline 7 & 6 & 0 & -0.775922 & -1.681339 & -0.358241 \\
\hline 8 & 6 & 0 & 0.775922 & -1.681339 & -0.358241 \\
\hline 9 & 6 & 0 & 1.454088 & -0.866940 & 0.748826 \\
\hline 10 & 1 & 0 & 2.000712 & 2.083104 & -0.841005 \\
\hline 11 & 1 & 0 & 0.000000 & 2.173678 & -2.135033 \\
\hline 12 & 1 & 0 & 2.075676 & 1.113972 & 1.336809 \\
\hline 13 & 1 & 0 & -2.075676 & 1.113972 & 1.336809 \\
\hline 14 & 1 & 0 & -2.000712 & 2.083104 & $4 \quad-0.841005$ \\
\hline 15 & 1 & 0 & -1.129233 & -1.210822 & $2 \quad 1.739316$ \\
\hline 16 & 1 & 0 & -2.530667 & -1.089483 & 0.708464 \\
\hline 17 & 1 & 0 & -1.134486 & -2.714399 & -0.266677 \\
\hline 18 & 1 & 0 & -1.146072 & -1.317554 & $4 \quad-1.322979$ \\
\hline 19 & 1 & 0 & 1.134486 & -2.714399 & - -0.266677 \\
\hline 20 & 1 & 0 & 1.146072 & -1.317554 & $4 \quad-1.322979$ \\
\hline 21 & 1 & 0 & 1.129233 & -1.210822 & $2 \quad 1.739316$ \\
\hline 22 & 1 & 0 & 2.530667 & -1.089483 & 0.708464 \\
\hline 23 & 1 & 0 & 0.000000 & 0.835574 & 1.071644 \\
\hline
\end{tabular}

SCF Done: $\mathrm{E}(\mathrm{RB}+\mathrm{HF}-\mathrm{LYP})=-351.279379911$ A.U. after 1 cycles Frequencies -- -1396.0269

Zero-point correction $=$ Thermal correction to Energy= Thermal correction to Enthalpy= Thermal correction to Gibbs Free Energy= Sum of electronic and zero-point Energies= Sum of electronic and thermal Energies= Sum of electronic and thermal Enthalpies= Sum of electronic and thermal Free Energies=

\subsection{7}

0.205296 (Hartree/Particle)

$$
0.213068
$$$$
0.214012
$$

0.172789

$-351.074084$

$-351.066312$

$-351.065367$

$-351.106591$ 
TS2

\#6-31 g* b3lyp freq nopop guess=read geom=check

Input orientation:

\begin{tabular}{|c|c|c|c|c|c|}
\hline \multirow{2}{*}{$\begin{array}{l}\text { Center } \\
\text { Number }\end{array}$} & \multirow{2}{*}{$\begin{array}{l}\text { Atomic } \\
\text { Number }\end{array}$} & \multirow{2}{*}{\multicolumn{2}{|c|}{$\begin{array}{l}\text { Atomic } \\
\text { Type }\end{array}$}} & \multicolumn{2}{|c|}{ Coordinates (Angstroms } \\
\hline & & & & X $\quad Y$ & Z \\
\hline 1 & 6 & 0 & 1.753420 & -1.200456 & 0.064466 \\
\hline 2 & 6 & 0 & 2.105189 & -0.037422 & -0.623250 \\
\hline 3 & 6 & 0 & 0.493338 & -1.301762 & 0.720917 \\
\hline 4 & 6 & 0 & 0.411857 & 1.403396 & 0.592332 \\
\hline 5 & 6 & 0 & 1.534799 & 1.194598 & -0.269604 \\
\hline 6 & 6 & 0 & -1.069058 & 1.624082 & 0.247705 \\
\hline 7 & 6 & 0 & -1.829341 & 0.641222 & -0.639795 \\
\hline 8 & 6 & 0 & -1.996602 & -0.753815 & -0.027832 \\
\hline 9 & 6 & 0 & -0.733991 & -1.607303 & -0.172040 \\
\hline 10 & 1 & 0 & 2.565432 & -1.886329 & 0.308210 \\
\hline 11 & 1 & 0 & 3.063763 & -0.007415 & -1.137671 \\
\hline 12 & 1 & 0 & 0.498309 & -1.935984 & 1.613770 \\
\hline 13 & 1 & 0 & 0.656092 & 2.197160 & 1.314482 \\
\hline 14 & 1 & 0 & 2.244298 & 2.022885 & -0.340163 \\
\hline 15 & 1 & 0 & -1.607506 & 1.709512 & 1.202684 \\
\hline 16 & 1 & 0 & -1.130889 & 2.624104 & -0.209376 \\
\hline 17 & 1 & 0 & -2.818776 & 1.077582 & -0.833728 \\
\hline 18 & 1 & 0 & -1.336102 & 0.554556 & -1.618508 \\
\hline 19 & 1 & 0 & -2.295122 & -0.670415 & 1.027390 \\
\hline 20 & 1 & 0 & -2.817896 & -1.274393 & -0.539350 \\
\hline 21 & 1 & 0 & -0.987232 & -2.661534 & 0.006171 \\
\hline 22 & 1 & 0 & -0.414664 & -1.552688 & -1.219429 \\
\hline 23 & 1 & 0 & 0.362632 & 0.027716 & 1.068124 \\
\hline
\end{tabular}

SCF Done: $\mathrm{E}(\mathrm{RB}+\mathrm{HF}-\mathrm{LYP})=-351.274183844$ A.U. after 1 cycles

Frequencies -- $1404.8746 \quad 91.8182 \quad 150.8190$

Zero-point correction $=\quad 0.204915$ (Hartree/Particle)

Thermal correction to Energy= $\quad 0.212675$

Thermal correction to Enthalpy= $\quad 0.213619$

Thermal correction to Gibbs Free Energy $=0.172768$

Sum of electronic and zero-point Energies $=\quad-351.069268$

Sum of electronic and thermal Energies $=\quad-351.061509$

Sum of electronic and thermal Enthalpies $=\quad-351.060565$

Sum of electronic and thermal Free Energies $=\quad-351.101416$ 
TS3

\# 6-31G* B3LYP FREQ GUESS=READ GEOM=CHECK NOPOP

Standard orientation:

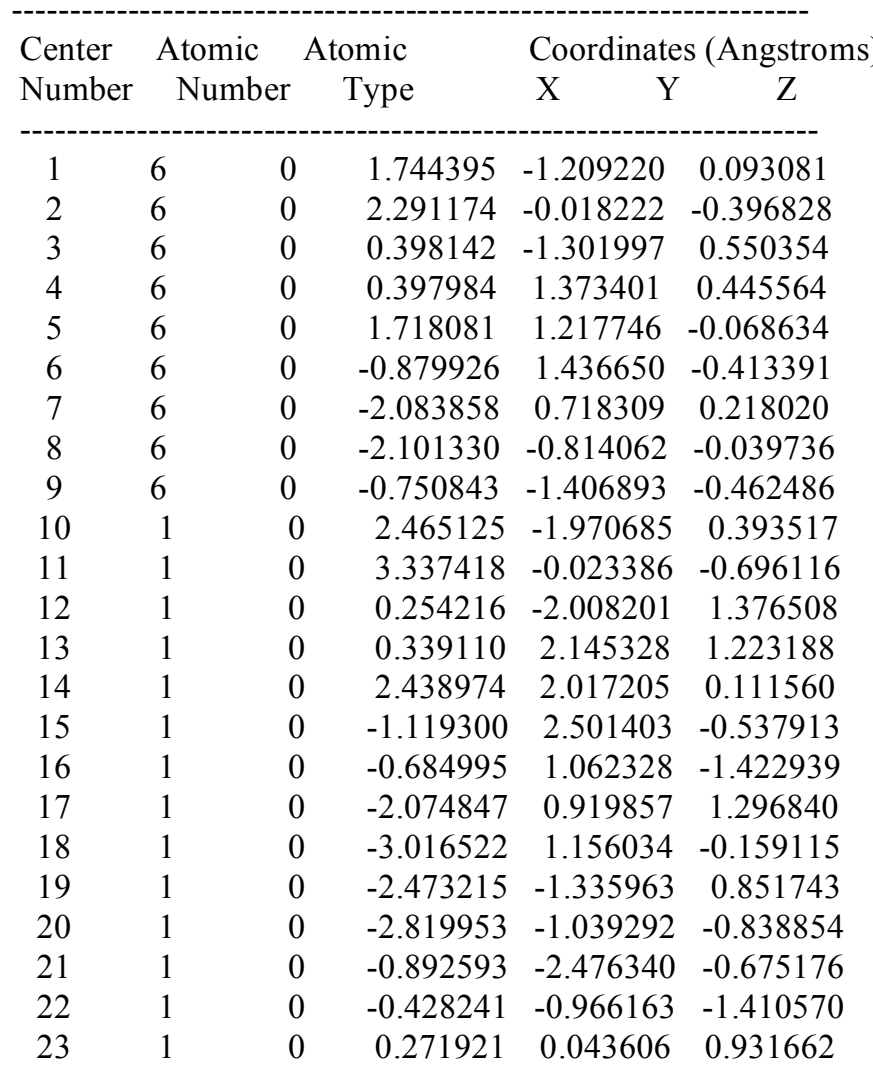

SCF Done: $\mathrm{E}(\mathrm{RB}+\mathrm{HF}-\mathrm{LYP})=-351.280236683$ A.U. after 1 cycles

Frequencies -- $-1399.4052 \quad 73.6793 \quad 203.0177$

Zero-point correction $=\quad 0.205274$ (Hartree/Particle)

Thermal correction to Energy $=\quad 0.213014$

Thermal correction to Enthalpy $=\quad 0.213958$

Thermal correction to Gibbs Free Energy $=\quad 0.173078$

Sum of electronic and zero-point Energies $=\quad-351.074963$

Sum of electronic and thermal Energies $=\quad-351.067223$

Sum of electronic and thermal Enthalpies= $\quad-351.066279$

Sum of electronic and thermal Free Energies $=\quad-351.107159$ 
Ground state conformational cis,cis-1,3-cyclononadiene isomers:
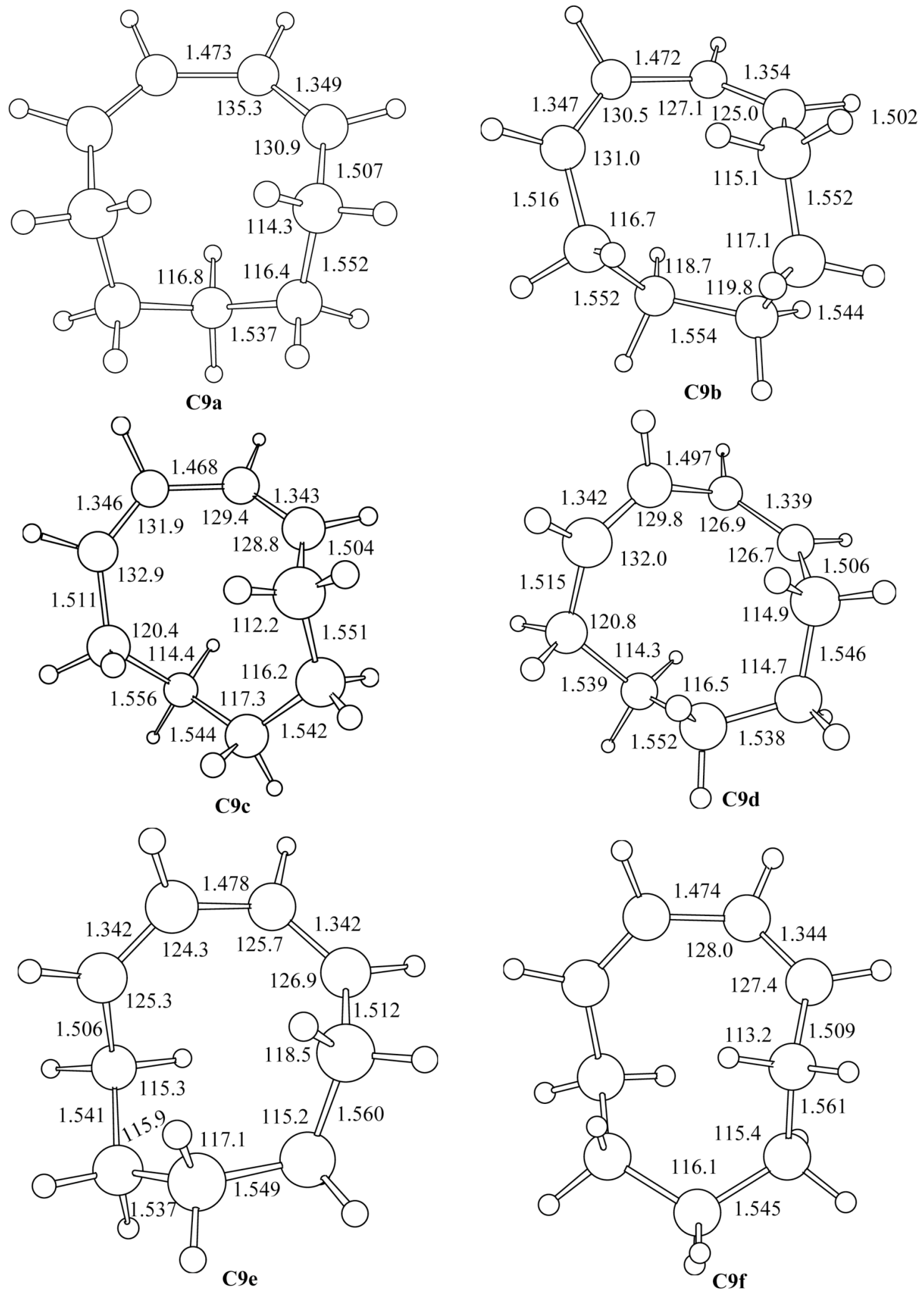
Transition state structures for isomerizations among conformers:

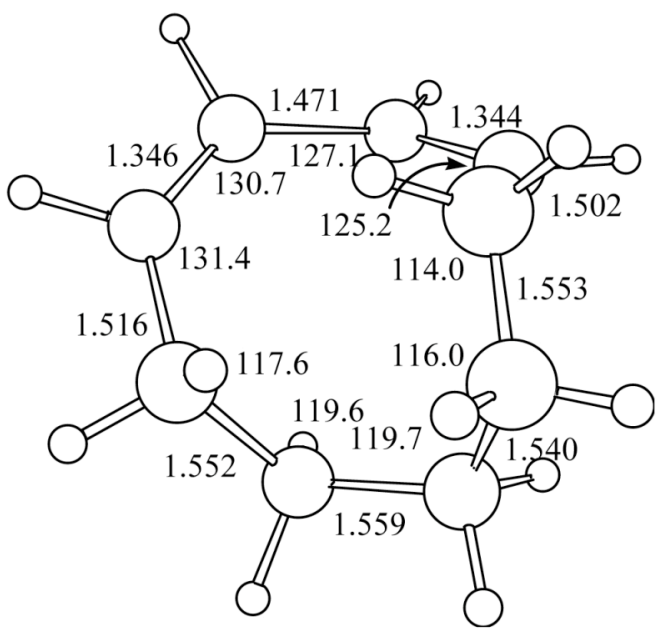

TSab

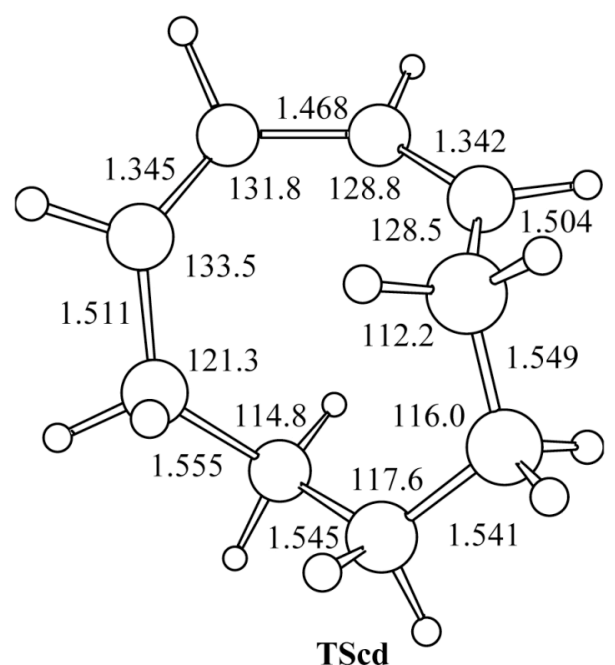

TSed

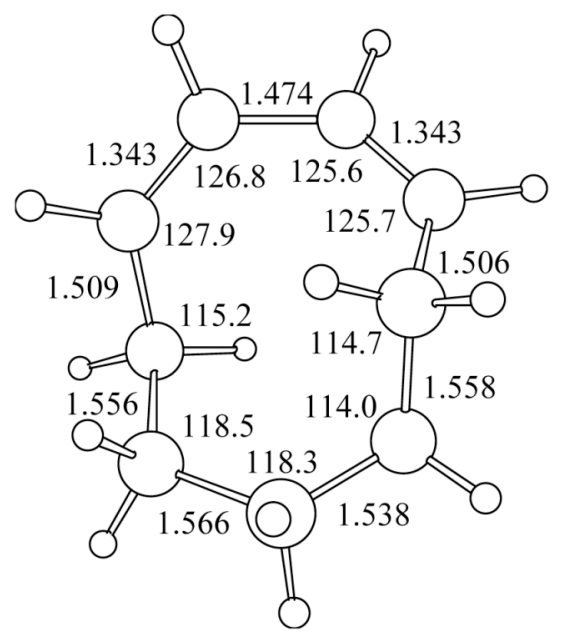

TSef

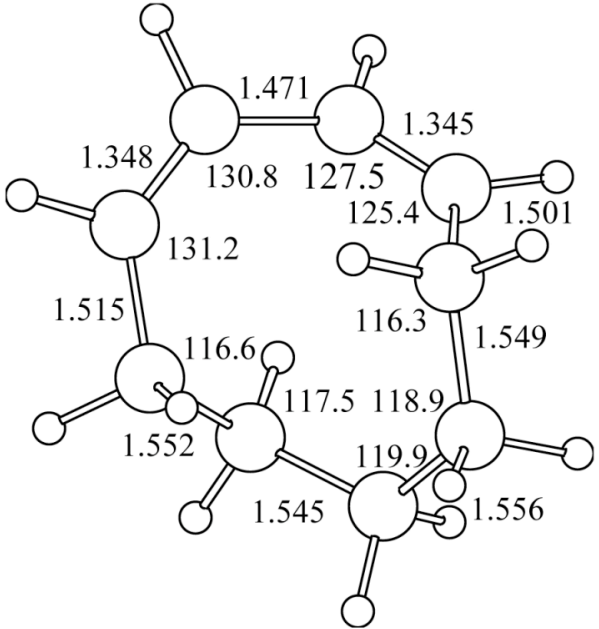

TSbe

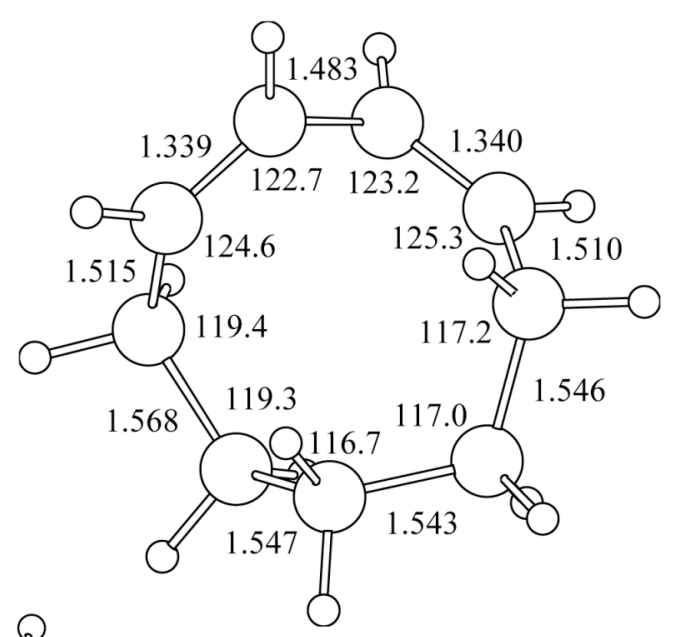

TSde 
Transition state structures for [1,5] hydrogen shifts:

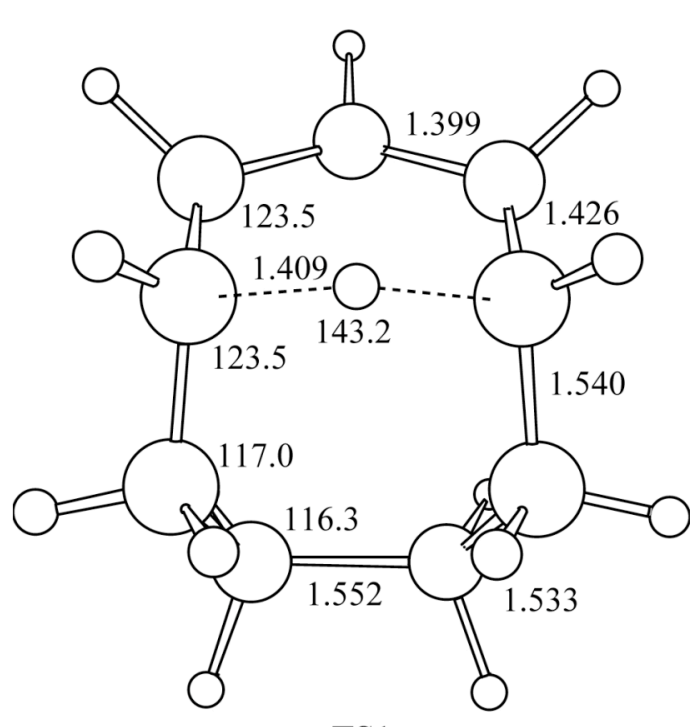

TS1

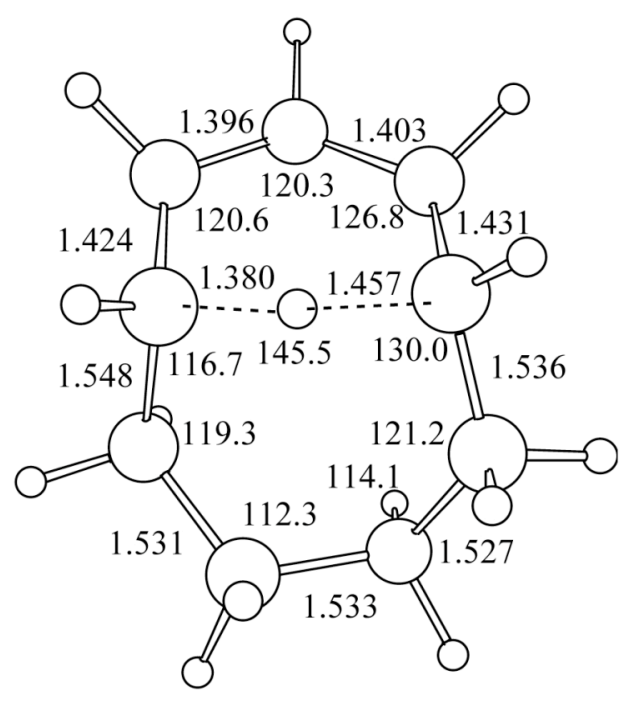

TS2

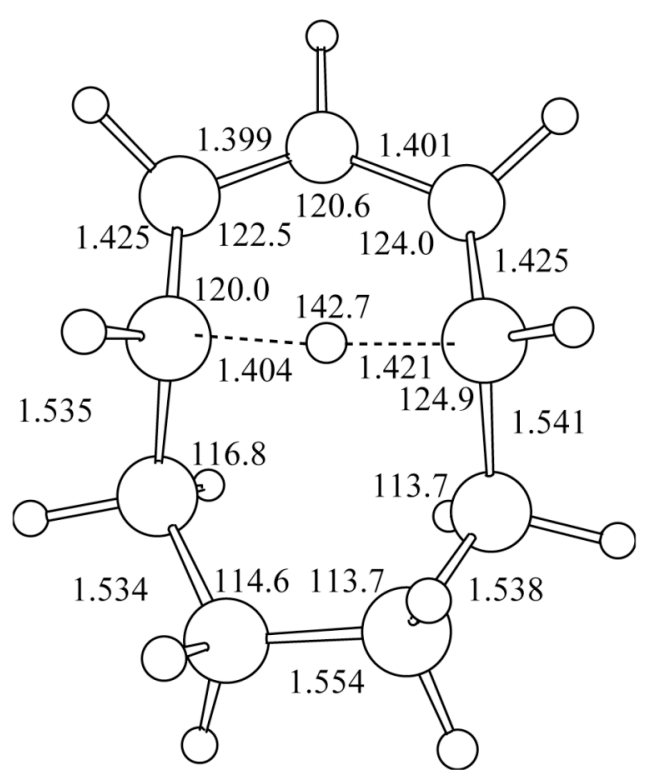

TS3 
Complete reference for Gaussian 98W:

Gaussian 98W: Frisch, M. J.; Trucks, G. W.; Schlegel, H. B.; Scuseria, G. E.; Robb, M. A.; Cheeseman, J. R.; Zakrzewski, V. G.; Montgomery, J. A., Jr.; Stratmann, R. E.; Burant, J. C.; Dapprich, S.; Millam, J. M.; Daniels, A. D.; Kudin, K. N.; Strain, M. C.; Farkas, O.; Tomasi, J.; Barone, V.; Cossi, M.; Cammi, R.; Mennucci, B.; Pomelli, C.; Adamo, C.; Clifford, S.; Ochterski, J.; Petersson, G. A.; Ayala, P. Y.; Cui, Q.; Morokuma, K.; Malick, D. K.; Rabuck, A. D.; Raghavachari, K.; Foresman, J. B.; Cioslowski, J.; Ortiz, J. V.; Stefanov, B. B.; Liu, G.; Liashenko, A.; Piskorz, P.; Komaromi, I.; Gomperts, R.; Martin, R. L.; Fox, D. J.; Keith, T.; Al-Laham, M. A.; Peng, C. Y.; Nanayakkara, A.; Gonzalez, C.; Challacombe, M.; Gill, P. M. W.; Johnson, B.; Chen, W.; Wong, M. W.; Andres, J. L.; Gonzalez, C.; Head-Gordon, M.; Replogle, E. S.; Pople, J. A. Gaussian, Inc.: Pittsburgh, PA, 1998. 